\title{
Odor Classification using Agent Technology
}

\author{
Sigeru Omatu ${ }^{\mathrm{a}}$, Tatsuyuki Wada ${ }^{\mathrm{a}}$, Pablo Chamoso ${ }^{\mathrm{b}}$ \\ ${ }^{\text {a }}$ Osaka Institute of Technology, Osaka, Japan \\ ${ }^{\mathrm{b}}$ Computer and Automation Department, University of Salamanca, Spain
}

KEYWORD

Odor sensing

Odor classification

Multi-agent system

Virtual organization

QCM sensor

\begin{abstract}
In order to measure and classify odors, Quartz Crystal Microbalance (QCM) can be used. In the present study, seven QCM sensors and three different odors are used. The system has been developed as a virtual organization of agents using an agent platform called PANGEA (Platform for Automatic coNstruction of orGanizations of intElligent Agents). This is a platform for developing open multi-agent systems, specifically those including organizational aspects. The main reason for the use of agents is the scalability of the platform, i.e. the way in which it models the services. The system models functionalities as services inside the agents, or as Service Oriented Approach (SOA) architecture compliant services using Web Services. This way the adaptation of the odor classification systems with new algorithms, tools and classification techniques is allowed.
\end{abstract}

\section{Introduction}

Ambient Intelligence has made huge technological strides in recent years [Want, R. et al., 1992], [Lim, C. H. et al., 2009]. Because of that is has acquired an important relevance in our daily lives [Bajo, J. et al., 2009], [Fonseca, S. P. et al., 2001], [Kowalczyk, R. et al., 2002]. Ambient Intelligence proposes three concepts when adapting technology to people's needs: ubiquitous computing, ubiquitous communication and intelligent user interfaces. In order to achieve these targets, it is necessary the development of new frameworks and models to allow info accessing, independently of the location. Wireless sensor networks [Ahmed, A. et al., 2006], [Lee, M. et al., 2007], [Hardy A. et al., 2010], provide an infrastructure able to distribute communications in dynamic environments by incrementing mobility and efficiency independently from the location. Sensor networks interconnect a large amount of sensors, as well as the info in the intelligent environment is managed. Many times the management of the information is done in a distributed way. Anyway, it is necessary to dispose of distributed systems capable to manage sensor networks in an efficient way. It is also necessary to dispose of elements with some degree of intelligence, acting both autonomously and coordinately with the distributed system, able to be embedded in the devices. A very suitable alternative to perform this type of systems is a multi-agent system. Several proposals to build smart environments combining multi-agent systems and sensor networks already exist [Carabelea, C. et al., 2003], [Fok, C. et al., 2009], [Meyer G. G. et al., 2009], [Kazanavicius, E. et al., 2009], [Tynam, R. et al., 2006], [Rogers, A. et al., 2009]. In order to support evolutional systems and facilitate their growth and runtime updates, new approaches are needed. Open environments dynamics is one of the reasons why the use of Virtual Organizations of Agents (VOs) has been encouraged. A VO [Ferber, J. et al., 2004], [Foster, I. et al., 2001], is an open system designed for grouping; it allows the collaboration among heterogeneous entities and provides a clear separation between the form and function defining their behavior. In any case, it is not possible to find an existing multiagent architecture able to work on the concept of virtual organizations and to provide agents capable of working with any kind of sensor or device. Different types of sensors - odor ones in this case - to classify odors based on the sensing data by using Quartz Crystal Microbalance (QCM) sensors are considered in the present article. QCM sensors are sensitive to odors and 
because of that, we are able to precisely measure the odor data. This paper focuses on the use of QCM sensors to try to classify various kinds of odors based on a neuronal network. In order to model the system, a virtual organization of agents, capable of providing high potential, is presented.

Using many QCM sensors, we will try to classify various kinds of odors based on a neural network. In order to model the system, a virtual organization of agents, capable of providing a greater number of possibilities is presented. Agents are connected to PANGEA platform [Zato, C. et al., 2012], a multi-agent platform designed on the basis of virtual organizations and focused on the creation of intelligent environments.

Over the last decade, odor-sensing systems (socalled electronic nose systems) have undergone an important development from a technical and commercial point of view. The EN refers to the capability of reproducing human sense of smell using sensor arrays and pattern recognition systems [Milke, 1995]. [Charumporn, B. et al., 2003] presents a type of EN system, based on a competitive neural network, to classify the various odors under the various densities of odors by using the learning vector quantization (LVQ) method. The odor data were measured by an odor sensor array made out of semiconductor metal oxide gas sensors (MOGSs). In particular, fourteen MOGSs of FIGARO Technology Ltd from Japan have been used. Two types of data for classification were considered in the experiment. The first one was a set of four kinds of tees and the second one was a set of five kinds of coffees with similar properties. The results of the classification of coffees and teas show a $96 \%$ and $89 \%$ accuracy respectively, which was much better than the results in [Fujinaka, T. et al., 2008] and [Omatu, S. et al., 2011].

The article is structured as follows. To start with PANGEA platform is described in Section 2, presenting in detail the structure of the virtual organization used in the odor classification case study. Both the platform and virtual organizations are evaluated on a case study consisting of an intelligent environment for odor recognition. Finally the results of the case study and the conclusions drawn from this research are presented.

\section{Case Study: Development of a VO for odor classification}

In this central section of the article, the integration of the system and the sensors used in the multi-agent architecture are presented, explaining the main concepts of QCM sensors. In addition, an overview of the odor sensing system and the measures of odor data used are described.

\subsection{Integration in a multi-agent platform (PANGEA)}

With the development of ubiquitous and distributed systems, it is interesting to have new agent platforms that facilitate the development of open agent-architectures able to deploy to any device. PANGEA [Zato, C. et al., 2012] is an agent platform that, based on organizational concepts, allows to model and implement all kinds of open systems, encouraging the sharing of resources and facilitating control of all nodes where the different agents are deployed.

It is considered essential to have control mechanisms to enable new devices to be included in a single platform where to easily be integrated, managed and monitored. In this case PANGEA, with its model of agents and organizations, provides the necessary features to behave as the base platform when developing a comprehensive system.

PANGEA has several agents that are automatically deployed when starting the platform operation to facilitate the control of the organization: OrganizationManager and OrganizationAgent in charge of the management of the organizations and suborganizations; InformationAgent: in charge of accessing the database containing all pertinent system information; ServiceAgent: in charge of recording and controlling the operation of services offered by the agents; NormAgent: in charge of the norms in the organization and CommunicationAgent: in charge of controlling communication among agents, and for recording the interaction between agents and organizations.

In the presented system, in addition to the intrinsic PANGEA agents, the organizations developed are the following: 
- Odor-recognition sensors organization. In this organization all agents belonging to an individual odor recognition system is deployed. Such agents may also be of different types (sensor type agents, interface type agent and identifier type agent).

- Sensor control central organization. In this organization the agent interface type is included representing each of the odorrecognition sensors organizations together with an agent of type adapter.

Communication in this case is restricted only to the existing agents in the same organization, plus the control agents that PANGEA architecture offers (as is the case of the Information Agent, for access to the database). Each type of agent is engaged in a well-defined task, as explained below:

- Sensor agent. It is exclusively dedicated to performing sensor readings and providing the latest value when an authorized agent requires such data.

- Identifier agent. Its function is to perform the necessary calculations for the identification of odors. It makes use of the ability to communicate with the agents of sensor type to require the data needed to perform these calculations.

- Interface agent. This kind of agent is present in the two types of virtual organizations cited. Is responsible for providing a communication link with the agents that, outside their own organization of odorrecognition sensors, are authorized to establish two-way communications using the appropriate communication format.

- Classifier agent. This agent performs services of classification that implement the algorithms described in the following section. To perform the classification, a Classification Method of Odor Data (e-BPNN) is used and implemented on the platform. We can say that the classifier agent could use new methods for the classification of odors by making the system scalable in terms of functionality.

- Adapter agent. This type of agent is in the central organization of sensor control. Its function is to try to correct the differences between the measurements of each of the associated sensors to the sensor type agents. Thus, a joint database between all recognition systems participating in the architecture is achieved, expanding the source of knowledge.

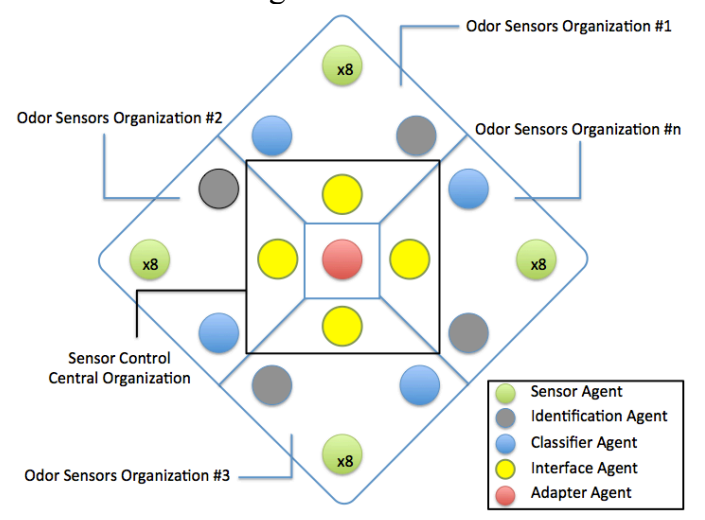

Figure 1: Structure of virtual organizations of the case study in PANGEA

\subsection{Algorithms: Classification Method of Odor Data (e- BPNN).}

We will show two types of neural networks, one is a multi-layered neural network based on error back-propagation method and another is a learning vector quantization (LVQ).

First, we will explain the multi-layered neural networks. In order to classify the odors, we adopt a three-layered neural network based on the error back-propagation method as shown in Fig. 2. The following steps give the error backpropagation algorithm, which is based on the gradient method.

- Step 1. Set the initial values of $w_{j i}, w_{k j}, \theta_{j}, \theta_{k}$, and $\eta(>0)$.

- $\quad$ Step 2. Specify the desired values of the output

$d_{k}, k=1,2, \cdots, K$ corresponding to the input data $x_{i}, i=1,2, \ldots, I$ in the input layer.

- $\quad$ Step 3. Calculate the outputs of the neurons in the hidden layer by

net $_{j}=\sum_{i=1}^{I} w_{j i} x_{i}-\theta_{j}, o_{j}=f\left(\right.$ net $\left._{j}\right), f(x)=\frac{1}{1+e^{-x}}$

- Step 4. Calculate the outputs of the neurons in the output layer by 
$n e t_{k}=\sum_{k=1}^{K} w_{k j i} O_{j}-\theta_{j}, O_{k}=f\left(n e t_{k}\right), f(x)=\frac{1}{1+e^{-x}}$

- Step 5. Calculate the error en and generalized errors by

$$
\begin{gathered}
e_{k}=d_{k}-O_{k} \\
\delta_{k}=\delta_{k} O_{k}\left(1-O_{k}\right) \\
\delta_{j}=\sum_{k=1}^{K} \delta_{k} w_{k j} O_{j}\left(1-O_{j}\right)
\end{gathered}
$$

- $\quad$ Step 6. Calculate by the following formula half of the sum of the squares of the errors in the output of all.

$$
\mathrm{E}=\frac{1}{2} \sum_{k=1}^{K} e_{k}^{2}
$$

- $\quad$ Step 7. If E is sufficiently small, exit the learning. Otherwise, modify the weight by the following equation:

$\Delta w_{k j} \equiv w_{k j}(t+1)-w_{k j}(t)=\eta \delta_{j} O_{j k} \quad \Leftarrow w_{k j}+\Delta w_{k j}$ $\Delta w_{j i} \equiv w_{j i}(t+1)-w_{j i}(t) \quad=\eta \delta_{i} O_{j} \quad \Leftarrow w_{j i}+\Delta w_{j i}$

- $\quad$ Step 8. Go to Step 3 .

Using the above recursive procedure, we can train the odor data.

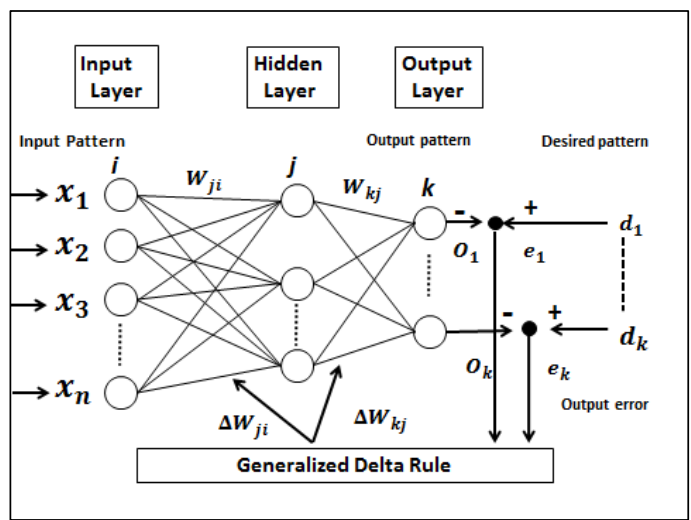

Figure 2: Three layered neural network with the error back-propagation

The neural network (Figure 2) consists of three layers, that is, an input layer $\mathrm{i}$, a hidden layer $\mathrm{j}$ and an output layer $\mathrm{k}$. When the input data $x_{i}, i=1,2, \ldots, I$, are applied in the input layer, we can obtain the output $O_{k}$ in the output layer which is compared with the desired value $d_{k}$ which is assigned in advance. If the error $e_{k}=d_{k}-O_{k}$ occurs, then the weighting coefficients $w_{j i}, w_{k j}$ are corrected such that the
The structure of LVQ is two layered, input layer and competitive layer as shown in Figure $\mathbf{3}$

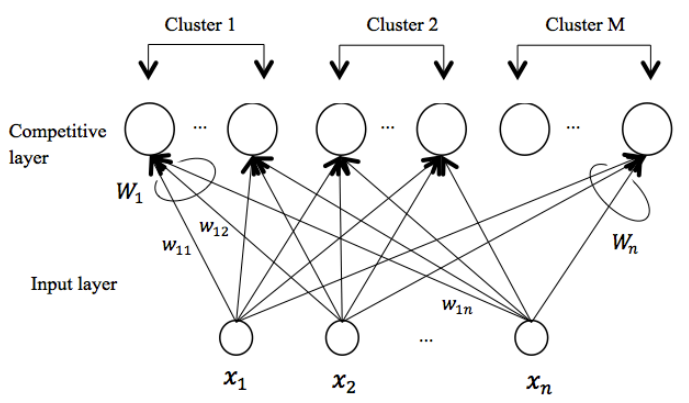

Figure 3: LVQ Structures

In order to classify the odors we adopt a twolayered neural network based on the learning vector quantization method as shown in Figure 3. Learning vector quantization is a supervised learning method for the purpose of pattern classification for the input data. The learning method is given by the following steps:

Step 1. Set the initial values of $w_{i j}$ $(j=1,2, \ldots, M, i=1,2, \ldots, n), T$, and $\alpha_{0}(>0)$ where $T$ is the total iteration number for learning, $n$ is the number of input, $M$ is the number of neurons in cluster $j$, and $\alpha_{0}$ is the initial value of the learning rate.

Step 2. First, It calculate proximity to the coupling coefficient vector WJ of the input vector $\mathrm{x}$ and neuron $\mathrm{j}$ in the sense of Euclidean distance. The neuron with the closest coupling coefficient in the sense of Euclidean distance in the competitive layer neuron is detected by following equation for the input pattern.

$$
\begin{gathered}
d_{j}=\left\|\mathrm{x}-w_{j}\right\|=\sqrt{\sum_{i=1}^{n}\left(x_{i}-w_{j i}\right)^{2}} \\
d_{c}=\left\|\mathrm{x}-w_{c}\right\|=\min _{j} d_{j}
\end{gathered}
$$

Step 3. If the input vector and winning neuron $\mathrm{c}$ belong to the same class, then change $w_{j}(t)$ by using the following equation:

where

$$
\begin{gathered}
w_{j}(t+1)=w_{j}(t)+\alpha(t)\left(x-w_{j}(t)\right), j=c \\
w_{j}(t+1)=w_{j}, j \neq c
\end{gathered}
$$

$$
\alpha(t)=\alpha_{0}\left(1-\frac{1}{T}\right) .
$$

If the input vector and neuron $\mathrm{c}$ belong to the different class, then change $w_{j}(t)$ by using the following equation: error becomes smaller based on the error backpropagation algorithm.

Next, we will show the LVQ in what follows:

Step 4. If $\mathrm{t}<\mathrm{T}$, go to Step 2 .

$$
\begin{gathered}
w_{j}(t+1)=w_{j}(t)-\alpha(t)\left(x-w_{j}(t)\right), j=c \\
w_{j}(t+1)=w_{j}, j \neq c .
\end{gathered}
$$

http://adcaj.usal.es 
Using the above recursive procedure, we can train the odor data.

\subsection{Principle of QCM sensors}

The QCM has been well known to provide very sensitive mass-measuring devices in nanogram levels. Synthetic polymer-coated QCMs have been studied as sensors for various gasses works as a chemical sensor. The QCM sensors are made by covering the surface with several kinds of a very thin membrane with about $1 \mathrm{~mm}$ as shown in Figure 4. The QCM sensor is integrated into a resonance circuit .If the odor molecules adsorb on the film, the oscillation frequency is reduced since the mass of the vibrator is changed.

So, the frequency (of the QCM) will change according to the deviation of the weight due to the adsorbed odor molecular (odorant). In this paper we have used the following materials as shown in Table 1 . The basic approach used here is a sol-gel method .The process is a wetchemical technique used for the fabrication of both glassy and ceramic materials. It gives the process of making a film by the following procedure.

MTMS (1): Trimethylsilane, ethanol, water, nitric acid. (2) It stirred added 2-ethyl acrylate (PFOEA) to a solution of 1 .

Here, $\quad$ HTMS: $\mathrm{CH}_{3} \mathrm{~S}_{\mathrm{i}}\left(\mathrm{OCH}_{3}\right)_{3} \quad$ and
PFOEA:F $\left(\mathrm{CF}_{2}\right)_{8} \mathrm{CH}_{2} \mathrm{CH}_{2}-\mathrm{OCOCH}=\mathrm{CH}_{2}$

\begin{tabular}{|c|c|}
\hline \multicolumn{2}{|c|}{ 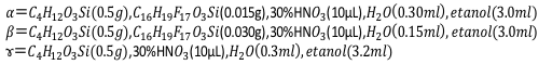 } \\
\hline \multicolumn{2}{|c|}{ Sensor num ber $M$ aterias of m ebrane } \\
\hline sensor1 & $\alpha$ \\
\hline sensor2 & $\beta$ \\
\hline sensor3 & $r$ \\
\hline sensor4 & $\alpha$ \\
\hline sensor5 & $r$ \\
\hline sensorf & $\alpha$ \\
\hline sensor 7 & $\beta$ \\
\hline
\end{tabular}

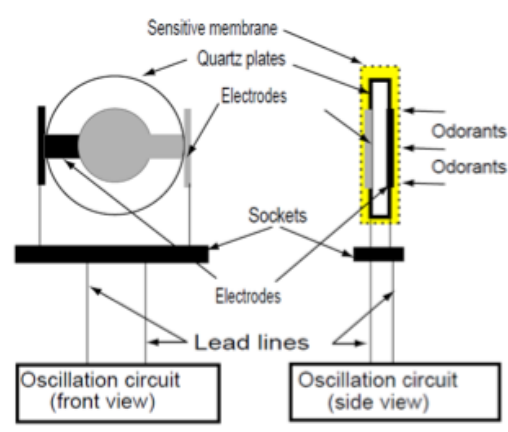

Figure 4: Principle of QCM sensor

The QCM sensor is integrated into a resonance circuit. If adsorbed odorant to the membrane of the sensor will make the weight of quartz plane. Therefore, oscillation frequency is reduced if adsorbed on the membrane. Thus, the original frequency of the crystal oscillation will become smaller according to the density of odorants.

\subsection{Odor Sensing Hardware System}

Generally, it is designed to detect some specific odor in electrical appliances such as an air purifier, a breath alcohol checker, and so on. Each of QCM membranes has its own characteristics in the response to different odors. When combining many QCM sensors together, the ability to detect the odor is increased. Odor sensing systems (so-called electronic nose (EN) systems) has been developed based on the concept of human olfactory system. Odor generation machines are using Permeater as shown in Figure 7.

Permeater can be generated continuously for a long time standard gas of many kinds (inorganic and organic gas) in the standard gas generator that generates a continuous standard of trace gas concentration. The combination of QCM sensors, listed in Table 1 , and is used as the olfactory receptors in the human nose. The odors used here are shown in Table 2. 


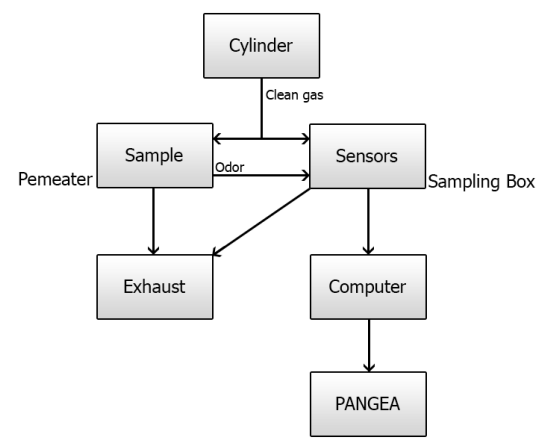

Figure 5: Odor sensing Systems

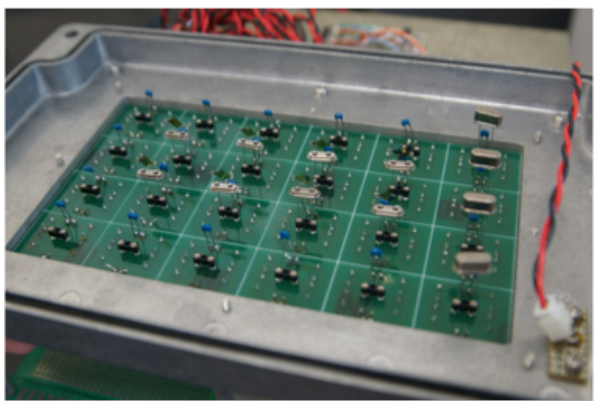

Figure 6: QCM sensors

First, add to odor generator by passing the dry air and then odor generated flows to sampling box. Then, reaction of the QCM sensor is accumulated into frequency counter as data. In addition, the gas is exhausted from the sampling box. The PC converts the data stored in the frequency counter into a text file.

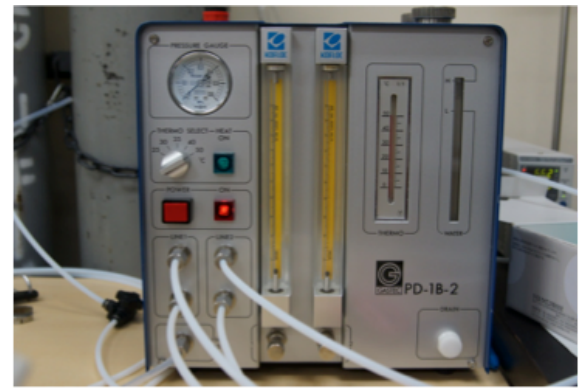

Figure 7: Permeater

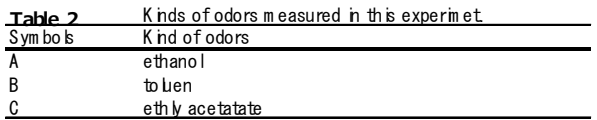

2.5. Measurement of Odor Data

We have measured four types of odors as shown in Table 2. To control the density of gases, we use the diffusion tubes. This is because it is possible to generate the gas at various concentrations by using a diffusion tube through Permeater. Odor data are measured for 900 [s]. They may include impulsive noises due to the typical phenomena of QCM sensors. To remove these impulsive noises we adopt a median filter, which replaces a value at a specific time by a median value among neighboring data around the specific time. In Figure $\mathbf{8}$ we show the measurement data for the symbol A where the horizontal axis is the measurement time and the vertical axis is the frequency deviation from the standard value $(20 \mathrm{M}[\mathrm{Hz}])$ after passing through a three-point median filter.

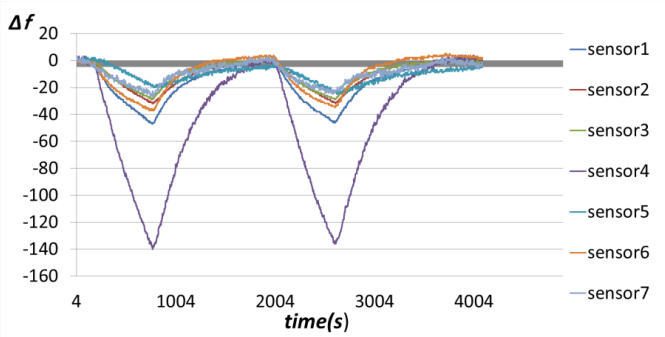

Figure 8: Measurement of odor data

Here, seven sensors are used and for nine hundred the data were measured. The maximum value for each sensor among seven sensors is selected as a feature value for the sensor. Therefore, we have seven sensor values for an odor and they will be used for classification.

\section{Conclusions and Results}

In order to classify the feature vector by using error-back propagation, we allocate the desired output for the input feature vector where it is seven-dimensional vector as shown in Table 3 since we have added the coefficient of variation to the usual feature vector to reduce the variations for odors. The training has been performed until the total error becomes less than or equal to $1 \times 10-2$ where $\eta=0.8$.

\begin{tabular}{|cccc|}
\hline Symbols & Output A & Output B & Output C \\
\hline A & 1 & 0 & 0 \\
\hline B & 0 & 1 & 0 \\
\hline C & 0 & 0 & 1 \\
\hline
\end{tabular}

Table 3: Training data set for ethanol (A), toluene (B) and ethyl acetate (C)

We have examined two algorithm, learning vector quantization and error back propagation 
.In learning vector quantization and error back propagation, the training sample number $\mathrm{P}^{\prime}=8$ and test sample number is three. By changing the training data set for 20 times and checked the classification accuracy for the test data samples. Thus, the total number of classification of 100 test samples is checked. The results are summarized in Table 4 and Table 5.

\begin{tabular}{|ccccc|}
\hline $\begin{array}{l}\text { Odor } \\
\text { data }\end{array}$ & A & B & C & Correct \\
\hline A & 100 & 0 & 0 & 100 \\
\hline B & 1 & 97 & 2 & 97 \\
\hline C & 4 & 1 & 95 & 95 \\
\hline
\end{tabular}

Table 4: Classification results for learning vector classification (LVQ)

\begin{tabular}{|c|c|c|c|c|}
\hline \multirow{2}{*}{$\begin{array}{l}\text { Odor } \\
\text { data }\end{array}$} & \multicolumn{4}{|c|}{ Classification results (88\%) } \\
\hline & A & B & $\mathrm{C}$ & Correct \\
\hline $\mathbf{A}$ & 86 & 14 & 0 & 86 \\
\hline B & 14 & 84 & 2 & 84 \\
\hline C & 2 & 3 & 95 & 95 \\
\hline
\end{tabular}

Table 4: Classification results for layered neural networks
We have presented the reliability of a new EN system designed from various kinds of QCM sensors. It has been shown that after training the neural network for each odor, we can classify the original odor from the mixed odors in case of two odors.

In addition, we have purpose and develop a multi-agent system which is able to increment the percentage of correct outputs and reduce the training time by sharing all the data between other similar odor detecting systems and correcting the error between their sensors. The multi-agent system implemented in PANGEA performs services of communication, control and management of information in a distributed and flexible way. In the case study presented, a classification method is implemented but thanks to the capabilities of PANGEA would be possible to extend the system using new methods for the classification of odors by making the system scalable in terms of functionality.

\section{$4 \quad$ References}

[Want, R. et al., 1992]

[Lim, C. H. et al., 2009]

[Bajo, J. et al., 2009]

[Fonseca, S. P. et al., 2001]

[Kowalczyk, R. et al., 2002]

[Ahmed, A. et al., 2006]

[Lee, M. et al., 2007]
R. Want, A. Hopper, V. Falcao, J. Gibbons, The Active Badge Location System. ACM Transactions on Information Systems, 10(1) (1992) 91102.

C. H. Lim, P, Anthony, L. C. Fan, Applying multi-agent system in a context aware. Borneo Science 24, (2009) 53-64.

J. Bajo, J. M. Corchado, Y. De Paz, J. F. De Paz, S. Rodríguez, Q. Martin, A. Abraham, SHOMAS: Intelligent Guidance and Suggestions in Shopping Centres. Applied Soft Computing. Elsevier Science, 9 (2) (2009) 851-862.

S. P. Fonseca, P. Martin, L. Griss, R. Letsinger, An Agent Mediated ECommerce Environment for the Mobile Shopper, Hewlett-Packard Laboratories, Technical Report, HPL-2001-157, 2001.

R. Kowalczyk, M. Ulieru, R. Unland, Integrating Mobile and Intelligent Agents in Advanced e-Commerce: A Survey. In Proceedings of the Agent Technologies, Infrastructures, Tools, and Applications for E-Services, NODe 2002, Lecture Notes in Computer Science, Vol. 2592, Springer, Berlin, 2002, pp. 295-313.

A. Ahmed, J. Ali, A. Raza, G. Abbas, Wired Vs Wireless Deployment Support For Wireless Sensor Networks, TENCON 2006. 2006 IEEE Region 10 Conference, 2006, pp. 1-3.

M. Lee, H. Yoe, Comparative Analysis and Design of Wired and Wireless Integrated Networks for Wireless Sensor Networks, Software Engineering Research, Management \& Applications, 2007. SERA

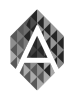


[Hardy A. et al., 2010]

[Carabelea, C. et al., 2003]

[Fok, C. et al., 2009]

[Meyer G. G. et al., 2009]

[Kazanavicius, E. et al., 2009]

[Tynam, R. et al., 2006]

[Rogers, A. et al., 2009]

[Ferber, J. et al., 2004]

[Foster, I. et al., 2001]

[Zato, C. et al., 2012]

[Milke, J. A.,1995]

[Charumporn, B. et al., 2003]

[Fujinaka, T. et al., 2008]

[Omatu, S. et al., 2011]
2007. 5th ACIS International Conference on, 2007, pp. 518-522.

A. Hardy, F. Bouhafs, M. Merabti, A Survey of Communication and Sensing for Energy Management of Appliances. International journal of advanced engineering sciences and technologies 3(2) (2010) 61-77.

C. Carabelea, O. Boissier, Multi-Agent Platforms on Smart Devices : Dream or Reality? In Proceedings of the SOC 2003, 2003 pp. 126-129.

C. Fok, G. Roman, C. Lu. Agilla, A Mobile Agent Middleware for Self-Adaptive Wireless Sensor Networks. ACM Transactions on Autonomous and Adaptive Systems (TAAS) 4(3) (2009) 1-26.

G. G. Meyer, K. Främling, J. Holmström, Intelligent Products: A survey, Computers in Industry, 60 (3) (2009) 137-148.

E. Kazanavicius, V. Kazanavicius, L. Ostaseviciute, Agent-based framework for embedded systems development in smart environments. In Proceedings of the 15th International Conference on Information and Software Technologies IT (2009) 194-200.

R. Tynam, A. Ruzzelli, G.M.P. O'Hare, A Methodology for the Deployment of Multi-Agent Systems on Wireless Sensor Networks. Multiagent and Grid Systems. (2006) 491-503.

A. Rogers, N.R. Jennings, D. Corkill, Agent Technologies for Sensor Networks. IEEE Intelligent Systems 24(2) (2009) 13-17.

J. Ferber, O. Gutknecht, F. Michel, From Agents to Organizations: an Organizational View of Multi-Agent Systems, in: P. Giorgini, J. Muller, J. Odell (Eds.), Agent-Oriented Software Engineering VI, LNCS Springer-Verlag. 2935, (2004) pp. 214-230.

I. Foster, C. Kesselman, S. Tuecke, The anatomy of the grid: Enabling scalable virtual organizations, Int. J. High Perform. Comput. Appl. 15 (3) (2001) 200-222.

C. Zato, G. Villarrubia, A. Sánchez, I. Barri, E. Rubión, A. Fernández, C. Rebate, J.A. Cabo, T. Álamos, J. Sanz, J. Seco, J., J. Bajo, J.M. Corchado, PANGEA - Platform for Automatic coNstruction of orGanizations of intElligent Agents. In Proceedings of the DCAI, Vol. 151, Springer, 2012, pp. 229-239.

J. A. Milke, "Application of Neural Networks for discriminating Fire Detectors". International Conference on Automatic Fire Detection, AUBE'95, 10th, Duisburg, Germany, pp. 213-222, 1995

Charumporn, B., Yoshioka, M., Fujinaka, T., \& Omatu, S. (2003, July). Identify household burning smell using an electronic nose system with artificial neural networks. In Computational Intelligence in Robotics and Automation, 2003. Proceedings. 2003 IEEE International Symposium on (Vol. 3, pp. 1070-1074). IEEE.

Fujinaka, T., Yoshioka, M., Omatu, S., \& Kosaka, T. (2008, September). Intelligent Electronic Nose Systems for Fire Detection Systems Based on Neural Networks. In Advanced Engineering Computing and Applications in Sciences, 2008. ADVCOMP'08. The Second International Conference on (pp. 73-76). IEEE.

Omatu S. and Yano M. (2011), "Intelligent Electronic Nose System Independent on Odor Concentration", International Symposium on Distributed Computing and Artificial Intelligence, Salamanca, Spain, pp. 1-9. 